\title{
On a generalized relaxed Saint-Venant principle
}

\author{
Marin Marin ${ }^{1 *}$, Ravi P. Agarwal ${ }^{2,3}$ and Dumitru Baleanu ${ }^{4,5}$
}

${ }^{\text {"Correspondence: }}$

m.marin@unitbv.ro

'Department of Mathematics and Computer Science, Transilvania

University of Brasov, Brasov, Romania

Full list of author information is available at the end of the article

\begin{abstract}
The goal of this paper is to obtain an extension of the relaxed Saint-Venant principle in order to cover the thermoelasticity of dipolar porous bodies.

According to this principle, for a finite time $t>0$, we identify a bounded domain $\mathcal{D}_{t}$ so that outside of this domain the displacement field $u_{i}$, the dipolar displacement field $\varphi_{j k}$, the temperature $\theta$, and the change in volume fraction $\phi$ do not generate disturbances.
\end{abstract}

MSC: 74A15; 74F05; 35Q74; 35Q79

Keywords: Dipolar bodies; Thermoelasticity; Voids; Relaxed Saint-Venant principle; Domain of influence

\section{Introduction}

There has been much written in recent years on the theory of thermoelastic bodies with pores. Our present study also deals with the thermoelasticity of body materials with vacuous pores or voids. It is known that the initiators of this theory were Nunziato and Cowin [1]. As it is well known, (see also [2, 3]) this theory allows the body to have an extra degree of freedom for a better characterization of its behavior from a mechanical point of view. So, the elastic body is the skeletal material and the voids of material are the interstices. The materials with porosity are of interest in geophysics (some geo-materials, for instance, soil or rocks) and artificially obtained granular materials.

The linear case, the basic theory of elastic porous bodies, was proposed by Cowin and Nunziato in [2]. This study includes a result of uniqueness and some considerations on the weak stability. After that, Iesan in [4] approached the basic results in thermoelasticity of porous bodies. Some generalized results were obtained for the theory of Cosserat solids with vacuous pores and the dipolar porous bodies $[5,6]$.

The origin of the theory of bodies which microstructure goes back to the papers of Eringen (see $[7,8])$. Then the studies dedicated to microstructure environments have gained significant importance in the last decade.

The dipolar structure occupies a privileged place between the theories that are dedicated to the microstructure. It is dedicated to eliminating the known contradictions of classical theory. First, it is known that the energy equation is of parabolic type and also the equation of energy does not contain any elastic term. As a consequence, under these conditions, the heat waves will propagate at an infinite speed. The importance of the dipolar structure of

(c) The Author(s) 2018. This article is distributed under the terms of the Creative Commons Attribution 4.0 International License (http://creativecommons.org/licenses/by/4.0/), which permits unrestricted use, distribution, and reproduction in any medium, provided you give appropriate credit to the original author(s) and the source, provide a link to the Creative Commons license, and indicate if changes were made. 
solids also derives from the interest shown for this structure by some of the outstanding researchers. We have to remember first of all the studies of Mindlin [9], Green and Rivlin [10], and Fried and Gurtin [11] which are very significant from this point of view. Other approaches to different aspects of the generalized bodies can be found in [12-20].

In the present study we first consider the basic equations and conditions of the mixed initial-boundary value problem in the context of thermoelasticity of dipolar bodies with voids. Next we define the concept of the domain of influence $\mathcal{D}_{t}$ regarding the data of our problem at some time $t>0$.

Inspired by the method used in the papers [21-23], we prove a domain of influence theorem in order to generalize the previous similar theorems.

In short, our result asserts that the solutions of the mixed initial-boundary value problem, in our context, vanishes outside $\mathcal{D}_{t}$ for some time $t>0$.

\section{Basic equations}

In the following we consider an elastic body in the general case of anisotropy. Such a material occupies a regular region $B$ from the Euclidian space $R^{3}$ and its border is assumed by a piecewise smooth surface denoted by $\partial B$. The closure of the domain $B$ is $\bar{B}$, and we have $\bar{B}=B \cup \partial B$.

We will use a system of Cartesian axes $O x_{i}(i=1,2,3)$ and adopt the Cartesian vector and tensor notation. The material time derivative will be highlighted by a superposed dot, while for the partial derivatives with respect to the spatial coordinates, we will use a comma followed by the respective subscript. Also, we adopt the summation rule (Einstein) on repeated indices.

When there is no likelihood of confusion, the spatial argument or/and the time argument of a function will be omitted. In order to describe the behavior of a dipolar body, we will use the following variables:

$$
u_{i}(t, X), \varphi_{i j}(t, X),(t, X) \in\left[0, t_{0}\right) \times B
$$

As such, the displacement vector field has components $u_{i}$ and the dipolar displacement tensor field has the components $\varphi_{i j}$.

With the help of the variables from (1), we can define the strain tensors of components $\varepsilon_{i j}, \gamma_{i j}$, and $\chi_{i j k}$ by means of the kinematic relations:

$$
2 \varepsilon_{i j}=u_{j, i}+u_{i, j}, \quad \gamma_{i j}=u_{j, i}-\varphi_{i j}, \quad \chi_{i j k}=\varphi_{i j, k} .
$$

In the theory of dipolar bodies we can define three stress tensors $\tau_{i j}$, $\eta_{i j}$, and $\mu_{i j k}$. These tensors are intrinsically linked to the above strain tensors by means of the constitutive equations:

$$
\begin{aligned}
\tau_{i j}= & A_{i j m n} \varepsilon_{m n}+G_{i j m n} \gamma_{m n}+F_{m n r i j} \chi_{m n r} \\
& +a_{i j} \phi+D_{i j k} \phi_{, k}-\alpha_{i j} \theta, \\
\eta_{i j}= & G_{i j m n} \varepsilon_{m n}+B_{i j m n} \gamma_{m n} \\
& +D_{i j m n} \chi_{m n r}+b_{i j} \phi+E_{i j k} \phi_{, k}-\beta_{i j} \theta,
\end{aligned}
$$




$$
\begin{aligned}
\mu_{i j k}= & F_{i j k m n} \varepsilon_{m n}+D_{m n i j k} \gamma_{m n}+C_{i j k m n r} \chi_{m n r} \\
& +c_{i j k} \phi+F_{i j k m} \phi_{, m}-\delta_{i j k} \theta .
\end{aligned}
$$

For the equilibrated stress vector $h_{i}$, the intrinsic equilibrated force $g$, the specific entropy $\eta$, and the heat flux vector, we will use the following constitutive equations:

$$
\begin{aligned}
& h_{i}=D_{m n i} \varepsilon_{m n}+E_{m n i} \gamma_{m n}+F_{m n r i} \chi_{m n r}+d_{i} \phi+g_{i j} \phi_{, j}-a_{i} \theta, \\
& g=-a_{i j} \varepsilon_{i j}-b_{i j} \gamma_{i j}-c_{i j k} \chi_{i j k}-\xi \phi-d_{i} \phi_{, i}+m \theta, \\
& \eta=\alpha_{i j} \varepsilon_{i j}+\beta_{i j} \gamma_{i j}+\delta_{i j k} \chi_{i j k}+m \phi+a_{i} \phi_{, i}+a \theta, \\
& q_{i}=k_{i j} \theta_{, j} .
\end{aligned}
$$

Equations that govern the theory of thermoelasticity of dipolar bodies with voids are the following (see, for instance, $[9,10]$ ):

- the equations of motion:

$$
\begin{aligned}
& \left(\tau_{i j}+\eta_{i j}\right)_{, j}+\varrho F_{i}=\varrho \ddot{u}_{i}, \\
& \mu_{i j k, i}+\eta_{j k}+\varrho M_{j k}=I_{k r} \ddot{\varphi}_{j r} ;
\end{aligned}
$$

- the equation of energy:

$$
\varrho T_{0} \dot{\eta}=q_{i, i}+\varrho r
$$

- the equation of the equilibrated forces:

$$
h_{i, i}+g+\varrho L=\varrho \kappa \ddot{\phi} .
$$

For the variation of the temperature and for the variation of the change in volume fraction, we will use the expressions

$$
\theta=T-T_{0}, \quad \phi=\sigma-\sigma_{0} .
$$

The meaning of the notations used in the above equations is the following:

$\varrho$-the constant mass density;

$\eta$-the specific entropy;

$T_{0}$ - the constant absolute temperature of the body in its reference state;

$I_{i j}$ - the coefficients of microinertia;

$\kappa$-the equilibrated inertia;

$u_{i}$-the components of displacement vector;

$\varphi_{j k}$-the components of dipolar displacement tensor;

$\sigma$-the volume distribution function which in the reference state is $\sigma_{0}$;

$\phi$-the change in volume fraction measured from the reference state;

$\theta$-the temperature variation measured from the reference temperature $T_{0}$;

$\varepsilon_{i j}, \gamma_{i j}, \chi_{i j k}$-the kinematic characteristics of the strain;

$\tau_{i j}, \eta_{i j}, \mu_{i j k}$ - the components of the stress tensors;

$h_{i}$-the components of the equilibrated stress vector;

$q_{i}$-the components of the heat flux vector; 
$F_{i}$-the components of the body forces;

$M_{j k}$-the components of the dipolar body forces;

$r$-the heat supply per unit time;

$g$-the intrinsic equilibrated force;

$L$ - the extrinsic equilibrated body force;

$A_{i j m n}, B_{i j m n}, \ldots, k_{i j}$-the elastic coefficients are characteristic functions of the material. Suppose the following symmetry relations take place:

$$
\begin{aligned}
& A_{i j m n}=A_{j i m n}=A_{m n i j}, \quad B_{i j m n}=B_{m n i j}, \quad a_{i j}=a_{j i}, \\
& d_{i j k}=d_{j i k}, \quad g_{i j}=g_{j i}, \quad C_{i j k m n r}=C_{m n r i j k}, \quad F_{i j k m n}=F_{i j k n m}, \\
& G_{i j m n}=G_{i j n m}, \quad k_{i j}=k_{j i} .
\end{aligned}
$$

Our mixed problem in the theory of thermoelasticity of dipolar bodies with vacuum pores will be complete if we add the known initial data:

$$
\begin{aligned}
& u_{i}(x, 0)=u_{i}^{0}(x), \quad \dot{u}_{i}(x, 0)=u_{i}^{1}(x), \\
& \varphi_{j k}(x, 0)=\varphi_{j k}^{0}(x), \quad \dot{\varphi}_{j k}(x, 0)=\varphi_{j k}^{1}(x), \\
& \phi(x, 0)=\phi^{0}(x), \quad \dot{\phi}(x, 0)=\phi^{1}(x), \\
& \theta(x, 0)=\theta^{0}(x), \quad x \in \bar{B},
\end{aligned}
$$

and also the given conditions to the limit

$$
\begin{array}{ll}
u_{i}=\bar{u}_{i} \quad \text { on } \partial B_{1} \times\left[0, t_{0}\right), & t_{i}=\bar{t}_{i} \quad \text { on } \partial B_{1}^{c} \times\left[0, t_{0}\right), \\
\varphi_{j k}=\bar{\varphi}_{j k} \quad \text { on } \partial B_{2} \times\left[0, t_{0}\right), & \mu_{j k}=\bar{\mu}_{j k} \quad \text { on } \partial B_{2}^{c} \times\left[0, t_{0}\right), \\
\phi=\bar{\phi} \quad \text { on } \partial B_{3} \times\left[0, t_{0}\right), & h=\bar{h} \quad \text { on } \partial B_{3}^{c} \times\left[0, t_{0}\right), \\
\theta=\bar{\theta} \quad \text { on } \partial B_{4} \times\left[0, t_{0}\right), & q=\bar{q} \quad \text { on } \partial B_{4}^{c} \times\left[0, t_{0}\right) .
\end{array}
$$

Here $\partial B_{1}, \partial B_{2}, \partial B_{3}$, and $\partial B_{4}$ with their respective complements $\partial B_{1}^{c}, \partial B_{2}^{c}$, $\partial B_{3}^{c}$, and $\partial B_{4}^{c}$ are subsets of $\partial B$ such that

$$
\begin{aligned}
& \partial B_{1} \cup \partial B_{1}^{c}=\partial B_{2} \cup \partial B_{2}^{c}=\partial B_{3} \cup \partial B_{3}^{c}=\partial B_{4} \cup \partial B_{4}^{c}=B, \\
& \partial B_{1} \cap \partial B_{1}^{c}=\partial B_{2} \cap \partial B_{2}^{c}=\partial B_{3} \cap \partial B_{3}^{c}=\partial B_{4} \cap \partial B_{4}^{c}=\emptyset .
\end{aligned}
$$

The fixed time $t_{0}$ from (10) can be infinite. Also, $u_{i}^{0}, u_{i}^{1}, \varphi_{j k}^{0}, \varphi_{j k}^{1}, \theta^{0}, \phi^{0}, \phi^{1}, \bar{u}_{i}, \bar{t}_{i}, \bar{\varphi}_{j k}, \bar{\mu}_{j k}$, $\bar{\phi}, \bar{\theta}, \bar{q}$, and $\bar{h}$ are known functions in their respective domains of definition.

The notations $t_{i}, \mu_{j k}, h$, and $q$ are used in (10) for the surface force tractions, which correspond to the displacement $u_{i}$, the dipolar displacement $\varphi_{j k}$, the equilibrated stress vector $h_{i}$, and the heat flux vector $q_{i}$ :

$$
t_{i} \equiv\left(\tau_{i j}+\eta_{i j}\right) n_{j}, \quad \mu_{j k} \equiv \mu_{i j k} n_{i}, \quad h \equiv h_{i} n_{i}, \quad q \equiv q_{i} n_{i},
$$

where $n_{i}$ are the components of the unit outward normal to $\partial B$. 
If we substitute the geometric equations (2) and the constitutive equations (3)-(4) into equations (5), (6), and (7), we arrive at the following system of field equations:

$$
\begin{aligned}
\varrho \ddot{u}_{i}= & {\left[\left(A_{i j m n}+G_{i j m n}\right) \varepsilon_{m n}+\left(G_{m n i j}+B_{i j m n}\right) \gamma_{m n}\right.} \\
& +\left(F_{m n r i j}+D_{i j m n r}\right) \chi_{m n r}+\left(a_{i j}+b_{i j}\right) \phi \\
& \left.\left.+\left(D_{i j k}+E_{i j k}\right) \phi_{, k}-\left(\alpha_{i j}+\beta_{i j}\right) \theta\right)\right]_{, j}+\varrho F_{i}, \\
I_{k r} \ddot{\varphi}_{j r}= & \left(F_{i j k m n} \varepsilon_{m n}+D_{m n i j k} \gamma_{m n}+C_{i j k m n r} \chi_{m n r}+c_{i j k} \phi\right. \\
& \left.+F_{i j k m} \phi_{, m}-\delta_{i j k} \theta\right)_{, i}+G_{j k m n} \varepsilon_{m n}+B_{j k m n} \gamma_{m n} \\
& +D_{j k m n r} \chi_{m n r}+b_{j k} \phi+D_{j k i} \phi_{, i}-\beta_{j k} \theta+\varrho M_{j k}, \\
\varrho \kappa \ddot{\phi}= & \left(D_{m n i} \varepsilon_{m n}+E_{m n i} \gamma_{m n}+F_{m n r i} \chi_{m n r}+d_{i} \phi+g_{i j} \phi_{, j}-a_{i} \theta\right)_{, i}+\varrho L \\
& -a_{i j} \varepsilon_{i j}-b_{i j} \gamma_{i j}-c_{i j k} \chi_{i j k}-\xi \phi-d_{i} \phi_{, i}+m \theta, \\
a \dot{\theta}= & \frac{1}{\varrho T_{0}}\left(k_{i j} \theta_{j, j}\right)_{, i}+\frac{1}{T_{0}} r-\beta_{i j} \dot{\varepsilon}_{i j}-\alpha_{i j} \dot{\gamma}_{i j}-\delta_{i j k} \dot{\chi}_{i j k}-m \dot{\phi}-a_{i} \dot{\phi}_{, i} .
\end{aligned}
$$

We must specify that from the inequality of producing entropy, we can deduce

$$
k_{i j} \theta_{, i} \theta_{, j} \geq 0 .
$$

An ordered array $\left(u_{i}, \varphi_{j k}, \phi, \theta\right)$ is a solution of the initial boundary value problem for the thermoelasticity of dipolar porous bodies in the cylinder $\Omega_{0}=B \times\left[0, t_{0}\right)$ if it satisfies the system of field differential equations (12) for all $(x, t) \in \Omega_{0}$, the conditions to the limit (10), and the initial data (9).

\section{Main result}

At the beginning of this section we will define the notion of domain of influence.

After that we will formulate and demonstrate an inequality that relates to the domain of influence. We have to say that this inequality is a generalization of similar inequality proposed in the papers [21-23]. This section and, in fact, our study ends with the demonstration of the theorem that establishes the domain of influence or, in other words, the relaxed Saint-Venant principle for the thermoelastic dipolar bodies with pores.

It is clear that the above mentioned results will be obtained if some conditions are met. The next assumptions on the properties of the material will help us in this endeavor:

(i) $\varrho>0, I_{i j}>0, \kappa>0, T_{0}>0, a>0$;

(ii)

$$
\begin{aligned}
A_{i j m n} & \xi_{i j} \xi_{m n}+2 G_{i j m n} \xi_{i j} \zeta_{m n}+B_{i j m n} \zeta_{i j} \zeta_{m n} \\
& +2 F_{m n r i j} \xi_{i j} v_{m n r}+2 D_{i j m n r} \zeta_{i j} v_{m n r}+C_{i j k m n r} v_{i j k} v_{m n r} \\
+ & 2 a_{i j} \xi_{i j} \omega+2 b_{i j} \zeta_{i j} \omega+2 c_{i j k} v_{i j k} \omega+2 D_{i j k} \xi_{i j} \omega_{k} \\
+ & 2 E_{i j k} \zeta_{i j} \omega_{k}+2 F_{i j k m} v_{i j k} \omega_{m}+2 d_{i} \omega_{i} \omega+\xi \omega^{2}+g_{i j} \omega_{i} \omega_{j} \\
\geq \alpha & \left.\alpha \xi_{i j} \xi_{i j}+\zeta_{i j} \zeta_{i j}+v_{i j k} v_{i j k}+\omega_{i} \omega_{i}+\omega^{2}\right),
\end{aligned}
$$

for all $\xi_{i j}=\xi_{j i}, \zeta_{i j}, v_{i j k}, \omega_{i}, \omega$;

(iii) $k_{i j} x_{i} x_{j} \geq \gamma x_{i} x_{i}$ for all $x_{i}$.

In these inequalities, $\alpha$ and $\gamma$ are conveniently chosen positive constants. 
We must note that these hypotheses are usual restrictions imposed in the mechanics of solids. For instance, hypothesis (iii) is a considerable strengthening of inequality (13) which, in turn, is a consequence of the inequality of entropy production.

Let us consider $V_{\alpha}(x)$, a smooth non-decreasing function, defined by

$$
V_{\alpha}(x)= \begin{cases}0, & \text { if } x \in(-\infty, 0], \\ 1, & \text { if } x \in[\alpha, \infty)\end{cases}
$$

for sufficiently small $\alpha>0$.

Function $V_{\alpha}$ is inspired by known Heaviside step function.

Using the function $V_{\alpha}$, for some fixed positive $\mathcal{R}_{1}$ and $t$, and for $d=\left|\mathbf{x}-\mathbf{x}_{0}\right|$, we define another useful function $G$ as follows:

$$
W(x, s)=V_{\alpha}\left(\frac{\mathcal{R}-d}{v}+t-s\right), \quad W: B \times[0, t] \rightarrow R,
$$

where $v$ is a positive constant having the dimension of velocity to be determined later and $\mathbf{x}_{0}$ is an arbitrary fixed point in $B$.

It is not difficult to find that the function $W(x, s)$ is a smooth function on the cylinder $B \times[0, t]$, and it vanishes outside the set

$$
\Sigma=\bigcup_{s \in[0, t]} \mathcal{S}\left[\mathbf{x}_{0}, \mathcal{R}+v(t-s)\right]
$$

Here $\mathcal{S}\left(\mathbf{x}_{0}, r\right)$ is a sphere defined by

$$
\mathcal{S}\left(\mathbf{x}_{0}, r\right)=\left\{\mathbf{x} \in R^{3}:\left|\mathbf{x}-\mathbf{x}_{0}\right|<r\right\} .
$$

The following inequality is a necessary step to obtain our main result.

Proposition 1 If the system of equations (12) admits a solution $\left(u_{i}, \varphi_{i j}, \phi, \theta\right)$ which satisfies the initial data (9) and the conditions to limit (10), then we have the following inequality:

$$
\begin{aligned}
{\left[\varrho \dot{u}_{i} \dot{u}_{i}\right.} & +I_{k r} \dot{\varphi}_{j r} \dot{\varphi}_{j k}+\varrho \kappa \dot{\phi}^{2}+a \theta^{2}+A_{i j m n} \varepsilon_{i j} \varepsilon_{m n} \\
& +2 G_{i j m n} \varepsilon_{i j} \gamma_{m n}+B_{i j m n} \gamma_{i j} \gamma_{m n}+2 F_{m n r i j} \varepsilon_{i j} \chi_{m n r} \\
& +2 D_{i j m n r} \gamma_{i j} \chi_{m n r}+C_{i j k m n r} \chi_{i j k} \chi_{m n r}+2 a_{i j} \varepsilon_{i j} \phi \\
& +2 b_{i j} \gamma_{i j} \phi+2 c_{i j k} \chi_{i j k} \phi+2 d_{i j k} \varepsilon_{i j} \phi_{, k}+2 e_{i j k} \gamma_{i j} \phi_{, k} \\
& \left.+2 f_{i j k m} \chi_{i j k} \phi_{, k}+2 d_{i} \phi \phi, i+g_{i j} \phi_{, i} \phi_{, j}+\xi \phi^{2}\right](x, s) \\
\geq & {\left[\varrho \dot{u}_{i} \dot{u}_{i}+I_{k r} \dot{\varphi}_{j r} \dot{\varphi}_{j k}+\varrho \kappa \dot{\phi}^{2}+a \theta^{2}\right.} \\
& \left.+\varepsilon_{i j} \varepsilon_{i j}+\gamma_{i j} \gamma_{i j}+\chi_{i j k} \chi_{i j k}+\phi^{2}+\phi_{, i} \phi_{, i}\right](x, s) .
\end{aligned}
$$

Proof The inequality is immediately obtained as a consequence of hypotheses (i) and (ii).

The following inequality uses inequality (16) and is the basis for obtaining the most important result of our paper. 
Theorem 1 If the system of equations (12) admits a solution $\left(u_{i}, \varphi_{i j}, \phi, \theta\right)$ which satisfies the initial data (9) and the data at the limit (10), then for any $t>0, \mathcal{R}>0$, and $\mathbf{x}_{0} \in B$, the following inequality holds:

$$
\begin{aligned}
& \int_{\Gamma\left[\mathbf{x}_{0}, \mathcal{R}\right]} P(\mathbf{x}, t) d V+\frac{1}{T_{0}} \int_{0}^{t} \int_{\Gamma\left[\mathbf{x}_{0}, \mathcal{R}+v(t-s)\right]} k_{i j} \theta_{, i} \theta_{, j} d V \\
& \leq \int_{\Gamma\left[\mathbf{x}_{0}, \mathcal{R}+v t\right]} P(\mathbf{x}, 0) d V \\
& \quad+\int_{0}^{t} \int_{\Gamma\left[\mathbf{x}_{0}, \mathcal{R}+v(t-s)\right]} \varrho\left[F_{i} \dot{u}_{i}+M_{j k} \dot{\varphi}_{j k}+L \dot{\phi}+\frac{1}{T_{0}} r \theta\right] d V d s \\
& \quad+\int_{0}^{t} \int_{\partial \Gamma\left[\mathbf{x}_{0}, \mathcal{R}+v(t-s)\right]}\left[\bar{t}_{i} \dot{u}_{i}+\bar{\mu}_{j k} \dot{\varphi}_{j k}+\bar{h} \dot{\phi}+\frac{1}{T_{0}} \bar{q} \theta\right] d A d s,
\end{aligned}
$$

where $\Gamma\left(\mathbf{x}_{0}, r\right)=\left\{\mathbf{x} \in B:\left|\mathbf{x}-\mathbf{x}_{0}\right|<r\right\}, \partial \Gamma\left(\mathbf{x}_{0}, r\right)=\left\{\mathbf{x} \in \partial B:\left|\mathbf{x}-\mathbf{x}_{0}\right|=r\right\}$.

Proof First, we must specify that the function $P(x, t)$ used in (17) is the potential energy and has the expression

$$
\begin{aligned}
P(x, s)= & \frac{1}{2}\left[\varrho \dot{u}_{i} \dot{u}_{i}+I_{k r} \dot{\varphi}_{j r} \dot{\varphi}_{j k}+\varrho \kappa \dot{\phi}^{2}+a \theta^{2}+C_{i j m n} \varepsilon_{i j} \varepsilon_{m n}\right. \\
& +2 G_{i j m n} \varepsilon_{i j} \gamma_{m n}+B_{i j m n} \gamma_{i j} \gamma_{m n}+2 F_{m n r i j} \varepsilon_{i j} \chi_{m n r} \\
& +2 D_{i j m n r} \gamma_{i j} \chi_{m n r}+A_{i j k m n r} \chi_{i j k} \chi_{m n r}+2 a_{i j} \varepsilon_{i j} \phi \\
& +2 b_{i j} \gamma_{i j} \phi+2 c_{i j k} \chi_{i j k} \phi 2 d_{i j k} \varepsilon_{i j} \phi_{, k}+2 e_{i j k} \gamma_{i j} \phi_{, k} \\
& \left.+2 f_{i j k m} \chi_{i j k} \phi_{, k}+2 d_{i} \phi \phi_{, i}+g_{i j} \phi_{, i} \phi_{, j}+\xi \phi^{2}\right](x, s) .
\end{aligned}
$$

Also, the kinetic energy is $K(x, s)$ which is a function defined by

$$
\begin{aligned}
K(x, s)= & \frac{1}{2}\left[\varrho \dot{u}_{i} \dot{u}_{i}+I_{k r} \dot{\varphi}_{j r} \dot{\varphi}_{j k}+\varrho \kappa \dot{\phi}^{2}+a \theta^{2}\right. \\
& \left.+\varepsilon_{i j} \varepsilon_{i j}+\gamma_{i j} \gamma_{i j}+\chi_{i j k} \chi_{i j k}+\phi^{2}+\phi_{, i} \phi_{, i}\right](x, s) .
\end{aligned}
$$

If we multiply both members of equation $(12)_{1}$ by $W \dot{u}_{i}$, we are led to the relation

$$
\begin{aligned}
\frac{1}{2} W \frac{d}{d t}\left(\varrho \dot{u}_{i} \dot{u}_{i}\right)= & \varrho W F_{i} \dot{u}_{i}+\left(W t_{i j} \dot{u}_{i}\right)_{, j}-W_{, j} t_{i j} \dot{u}_{i} \\
& -W\left(A_{i j m n} \varepsilon_{m n}+B_{i j m n} \gamma_{m n}+B_{i j} \phi+D_{i j k} \phi_{, k}-\beta_{i j} \theta\right) \dot{u}_{i, j} .
\end{aligned}
$$

By analogy, we multiply both members of equation $(12)_{2}$ by $W \dot{\varphi}_{j k}$, so that we obtain

$$
\begin{aligned}
\frac{1}{2} W \frac{d}{d t}\left(I_{k r} \dot{\varphi}_{j r} \dot{\varphi}_{j k}\right)= & \varrho G M_{j k} \dot{\varphi}_{j k}+\left(W \mu_{j k} \dot{\varphi}_{j k}\right)_{, j}-W_{, j} \mu_{j k} \dot{\varphi}_{j k} \\
& -W\left(B_{m n i j} \varepsilon_{m n}+C_{i j m n} \gamma_{m n}+C_{i j} \phi+E_{i j k} \phi_{, k}-\alpha_{i j} \theta\right) \dot{\varphi}_{j k, j} \\
& +\varepsilon_{i j k}\left(A_{j k m n} \varepsilon_{m n}+B_{j k m n} \gamma_{m n}+B_{j k} \phi+D_{j k m} \phi_{, m}-\beta_{j k} \theta\right) \dot{\varphi}_{j k} .
\end{aligned}
$$


Now, we multiply both members of equation $(12)_{3}$ by $W \dot{\phi}$, so we deduce that

$$
\begin{aligned}
\frac{1}{2} W \frac{d}{d t}\left(\varrho \kappa \dot{\phi}^{2}\right)= & \varrho W L \dot{\phi}+\left(W h_{i} \dot{\phi}\right)_{, i}-W_{, i} h_{i} \dot{\phi} \\
& -W\left(A_{i j} \phi_{, j} \dot{\phi}_{, i}+D_{m n i} \varepsilon_{m n} \dot{\phi}_{, i}+E_{m n i} \gamma_{m n} \dot{\phi}_{, i}+d_{i} \phi \dot{\phi}_{, i}-a_{i} \theta \dot{\phi}_{, i}\right) \\
& -W\left(B_{i j} \varepsilon_{i j} \dot{\phi}+C_{i j} \gamma_{i j} \dot{\phi}+\xi \phi \dot{\phi}+d_{i} \phi_{, i} \dot{\phi}-m \theta \dot{\phi}\right) .
\end{aligned}
$$

Finally, if we multiply both members of equation $(12)_{4}$ by $W \theta$, we are led to

$$
\begin{aligned}
\frac{1}{2} W \frac{d}{d t}\left(a \theta^{2}\right)= & \frac{1}{T_{0}} W r \theta+\frac{1}{\varrho T_{0}}\left[\left(W \theta q_{i}\right)_{, i}-W{ }_{i} \theta q_{i}\right] \\
& -\frac{1}{\varrho T_{0}} W k_{i j} \theta_{i} \theta_{, j}-W\left(\beta_{i j} \theta \dot{\varepsilon}_{i j}+\alpha_{i j} \theta \dot{\gamma}_{i j}+m \theta \dot{\phi}+a_{i} \theta \dot{\phi}_{, i}\right) .
\end{aligned}
$$

Summing up equations (20), (21), (22), and (23) term by term results in

$$
\begin{aligned}
\frac{1}{2} W & \frac{d}{d t}\left(\varrho \dot{u}_{i} \dot{u}_{i}+I_{k r} \dot{\varphi}_{j r} \dot{\varphi}_{j k}+\varrho \kappa \dot{\phi}^{2}+a \theta^{2}\right) \\
= & \varrho W F_{i} \dot{u}_{i}+\varrho W M_{j k} \dot{\varphi}_{j k} \\
& +\varrho W L \dot{\phi}+\frac{1}{T_{0}} W r \theta+W\left(t_{i j} \dot{u}_{i}+\mu_{j k} \dot{\varphi}_{j k}+h_{j} \dot{\phi}+\frac{1}{\varrho T_{0}} \theta q_{j}\right)_{, j} \\
& -W\left[A_{i j m n} \varepsilon_{m n} \dot{\varepsilon}_{i j}+B_{i j m n}\left(\varepsilon_{m n} \dot{\gamma}_{i j}+\dot{\varepsilon}_{m n} \gamma_{i j}\right)+C_{i j m n} \gamma_{m n} \dot{\gamma}_{i j}\right. \\
& +B_{i j}\left(\dot{\varepsilon}_{i j} \phi+\varepsilon_{i j} \dot{\phi}\right)+C_{i j}\left(\dot{\gamma}_{i j} \phi+\gamma_{i j} \dot{\phi}\right)+D_{i j k}\left(\varepsilon_{i j} \dot{\phi}_{, k}+\dot{\varepsilon}_{i j} \phi_{, k}\right) \\
& \left.+E_{i j k}\left(\gamma_{i j} \dot{\phi}_{, k}+\dot{\gamma}_{i j} \phi_{, k}\right)+d_{i}\left(\phi \dot{\phi}_{, i}+\dot{\phi} \phi_{, i}\right)+A_{i j} \phi_{, i} \dot{\phi}_{, j}+\xi \phi \dot{\phi}\right] \\
& -W_{, j} t_{i j} \dot{u}_{i}-W_{, j} \mu_{j k} \dot{\varphi}_{j k}-W_{, i} h_{i} \dot{\phi}-\frac{1}{\varrho T_{0}} W_{, i} q_{i} \theta-\frac{1}{\varrho T_{0}} W k_{i j} \theta_{, i} \theta_{, j} .
\end{aligned}
$$

Clearly, relation (24) can be rewritten in the following form:

$$
\begin{aligned}
\frac{1}{2} W & \frac{d}{d t}\left(\varrho \dot{u}_{i} \dot{u}_{i}+I_{k r} \dot{\varphi}_{j r} \dot{\varphi}_{j k}+\varrho \kappa \dot{\phi}^{2}+a \theta^{2}+A_{i j m n} \varepsilon_{m n} \varepsilon_{i j}+2 B_{i j m n} \gamma_{m n} \varepsilon_{i j}\right. \\
& +C_{i j m n} \gamma_{m n} \gamma_{i j}+2 B_{i j} \varepsilon_{i j} \phi+2 C_{i j} \gamma_{i j} \phi+2 D_{i j k} \varepsilon_{i j} \phi_{, k} \\
& \left.+2 E_{i j k} \gamma_{i j} \phi_{, k}+2 d_{i} \phi \phi_{, i}+A_{i j} \phi_{, i} \phi_{, j}+\xi \phi^{2}\right) \\
= & \varrho W\left(F_{i} \dot{u}_{i}+M_{j k} \dot{\varphi}_{j k}+\varrho L \dot{\phi}+\frac{1}{T_{0}} r \theta\right) \\
& +W\left(t_{i j} \dot{u}_{i}+\mu_{j k} \dot{\varphi}_{j k}+h_{j} \dot{\phi}+\frac{1}{\varrho T_{0}} \theta q_{j}\right)_{, j} \\
& -W_{, j} t_{i j} \dot{u}_{i}-W_{, j} \mu_{j k} \dot{\varphi}_{j k}-W_{, i} h_{i} \dot{\phi}-W_{, i} \frac{1}{\varrho T_{0}} \theta q_{i}-\frac{1}{\varrho T_{0}} k_{i j} \theta_{, i} \theta_{, j},
\end{aligned}
$$

or, equivalently,

$$
\frac{1}{2} W \dot{U}+\frac{1}{\varrho T_{0}} k_{i j} \theta_{, i} \theta_{, j}=W\left(\varrho F_{i} \dot{u}_{i}+\varrho M_{j k} \dot{\varphi}_{j k}+\varrho L \dot{\phi}+\frac{1}{T_{0}} \varrho r \theta\right)
$$




$$
\begin{aligned}
& +W\left(t_{i j} \dot{u}_{i}+\mu_{j k} \dot{\varphi}_{j k}+h_{j} \dot{\phi}+\frac{1}{\varrho T_{0}} \theta q_{j}\right)_{, j} \\
& -W_{, j}\left(t_{i j} \dot{u}_{i}+\mu_{j k} \dot{\varphi}_{j k}+h_{j} \dot{\phi}+\frac{1}{\varrho T_{0}} \theta q_{j}\right) .
\end{aligned}
$$

We now integrate on cylinder $B \times[0, t]$ both members of equality (26), and afterwards we use the divergence theorem and the boundary conditions (10), so we get

$$
\begin{aligned}
\int_{B} W U(x, t) d V+\frac{1}{\varrho T_{0}} \int_{0}^{t} \int_{B} W k_{i j} \theta_{, i} \theta_{, j} d V d s \\
=\int_{B} W U(x, 0) d V \\
\quad+\int_{0}^{t} \int_{\partial B} W\left(\bar{t}_{i} \dot{u}_{i}+\bar{\mu}_{j k} \dot{\varphi}_{j k}+\bar{h} \dot{\phi}+\frac{1}{\varrho T_{0}} \bar{q} \theta\right) d V d s \\
\quad+\int_{0}^{t} \int_{B} \varrho W\left(F_{i} \dot{u}_{i}+M_{j k} \dot{\varphi}_{j k}+L \dot{\phi}+\frac{1}{T_{0}} r \theta\right) d V d s \\
\quad+\int_{0}^{t} \int_{B} \dot{W} U(x, s) d V d s-\int_{0}^{t} \int_{B} W_{, j}\left(t_{i j} \dot{u}_{i}+\mu_{j k} \dot{\varphi}_{j k}+h_{j} \dot{\phi}+\frac{1}{\varrho T_{0}} q_{j} \theta\right) d V d s .
\end{aligned}
$$

Considering definition (14) of the function $W$, it is not difficult to find that

$$
\begin{aligned}
\mid- & W_{, j} t_{i j} \dot{u}_{i}-W_{, j} \mu_{j k} \dot{\varphi}_{j k}-W_{, i} h_{i} \dot{\phi}-\frac{1}{\varrho T_{0}} W_{, i} q_{i} \theta \mid \\
= & \left|\frac{1}{v} V_{\alpha}^{\prime} \frac{x_{j}}{\mathbf{r}} t_{i j} \dot{u}_{i}+\frac{1}{c} V_{\alpha}^{\prime} \frac{x_{j}}{\mathbf{r}} \mu_{j k} \dot{\varphi}_{j k}+\frac{1}{c} V_{\alpha}^{\prime} \frac{x_{i}}{\mathbf{r}} h_{i} \dot{\phi}+\frac{1}{c \varrho T_{0}} V_{\alpha}^{\prime} \frac{x_{i}}{\mathbf{r}} q_{i} \theta\right| \\
= & \mid \frac{1}{v} V_{\alpha}^{\prime} \frac{1}{\mathbf{r}}\left[\left(A_{i j m n} \varepsilon_{m n} x_{j}+B_{i j m n} \gamma_{m n} x_{j}+B_{i j} \phi x_{j}+D_{i j k} \phi_{, k} x_{j}-\beta_{i j} \theta x_{j}\right) \dot{u}_{i}\right. \\
& +\left(B_{m n i j} \varepsilon_{m n} x_{j}+C_{i j m n} \gamma_{m n} x_{j}+C_{i j} \phi x_{j}+E_{i j k} \phi_{, k} x_{j}-\beta_{i j} \theta x_{j}\right) \dot{\varphi}_{j k} \\
& \left.+\left(D_{m n i} \varepsilon_{m n} x_{i}+E_{m n i} \gamma_{m n} x_{i}+A_{i j} \phi_{, j} x_{i}+d_{i} \phi x_{i}-a_{i} \theta x_{i}\right) \dot{\phi}+\frac{1}{\varrho T_{0}} k_{i j} \theta \theta_{, j} \theta x_{i}\right],
\end{aligned}
$$

where

$$
V_{\alpha}^{\prime}=\frac{d V_{\alpha}}{d \mathbf{r}}
$$

The elementary arithmetic-geometric mean inequality

$$
a b \leq \frac{1}{2}\left(\frac{a^{2}}{p^{2}}+b^{2} p^{2}\right)
$$

is now used to the last terms of relation (28). Thus, we can choose some suitable parameters $p$ and can find $v$ such that

$$
\left|-W_{, j} t_{i j} \dot{u}_{i}-W_{, j} \mu_{j k} \dot{\varphi}_{j k}-W_{, i} h_{i} \dot{\phi}-\frac{1}{T_{0}} W_{, i} q_{i} \theta\right| \leq V_{\alpha}^{\prime} K(x, s),
$$


and also

$$
\begin{aligned}
& \int_{0}^{t} \int_{B} \dot{W} U(x, s) d V d s-\int_{0}^{t} \int_{B}\left(W_{, j} t_{i j} \dot{u}_{i}+W_{, j} \mu_{j k} \dot{\varphi}_{j k}+W_{, i} h_{i} \dot{\phi}+\frac{1}{T_{0}} W_{, i} q_{i} \theta\right) d V d s \\
& \quad \leq \int_{0}^{t} \int_{B} V_{\alpha}^{\prime}(x, s)[K(x, s)-U(x, s)] d V d s \leq 0 .
\end{aligned}
$$

Considering inequality (31), from equation (27) we are led to

$$
\begin{aligned}
\int_{B} W U(x, t) d V+\frac{1}{T_{0}} \int_{0}^{t} \int_{B} W k_{i j} \theta_{i} \theta_{, j} d V d s \\
\leq \int_{B} W U(x, 0) d V \\
\quad+\int_{0}^{t} \int_{B} \varrho W\left(F_{i} \dot{u}_{i}+M_{j k} \dot{\varphi}_{j k}+L \dot{\phi}+\frac{1}{\varrho^{2} T_{0}} r \theta\right) d V d s \\
\quad+\int_{0}^{t} \int_{\partial B} W\left(\bar{t}_{i} \dot{u}_{i}+\bar{\mu}_{j k} \dot{\varphi}_{j k}+\bar{h} \dot{\phi}+\frac{1}{\varrho T_{0}} \bar{q} \theta\right) d V d s .
\end{aligned}
$$

We will pass to the limit as $\alpha \rightarrow 0$ into relation (32), so that we deduce that $W$ tends boundedly to the characteristic function of the set $\Sigma$ (before (15)) and, as a consequence, we obtain inequality (17) and the proof of Theorem 1 is complete.

We will use previous estimates from Proposition 1 and Theorem 1 to demonstrate the basic result of the present study, namely an extension of the relaxed Saint-Venant principle or, in other words, a generalized theorem of the domain of influence.

We will denote by $B(t)$ the set of all points $\in \bar{B}$ having the following properties:

(1) if $x \in B$ then $u_{i}^{0} \neq 0$ or $u_{i}^{1} \neq 0$ or $\varphi_{j k}^{0} \neq 0$ or $\varphi_{j k}^{1} \neq 0$ or $\phi^{0} \neq 0$ or $\phi^{1} \neq 0$ or $\theta^{0} \neq 0$ or

$\exists \tau \in[0, t]$ such that $F_{i}(x, \tau) \neq 0$ or $M_{i}(x, \tau) \neq 0$ or $L(x, \tau) \neq 0$ or $r(x, \tau) \neq 0$;

(2) if $x \in \partial B_{1}$ then $\exists \tau \in[0, t]$ such that $\bar{u}_{i}(x, \tau) \neq 0$;

(3) if $x \in \partial B_{1}^{c}$ then $\exists \tau \in[0, t]$ such that $\bar{t}_{i}(x, \tau) \neq 0$;

(4) if $x \in \partial B_{2}$ then $\exists \tau \in[0, t]$ such that $\bar{\varphi}_{j k}(x, \tau) \neq 0$;

(5) if $x \in \partial B_{2}^{c}$ then $\exists \tau \in[0, t]$ such that $\bar{\mu}_{j k}(x, \tau) \neq 0$;

(6) if $x \in \partial B_{3}$ then $\exists \tau \in[0, t]$ such that $\bar{\phi}(x, \tau) \neq 0$;

(7) if $x \in \partial B_{3}^{c}$ then $\exists \tau \in[0, t]$ such that $\bar{h}(x, \tau) \neq 0$;

(8) if $x \in \partial B_{4}$ then $\exists \tau \in[0, t]$ such that $\bar{\theta}(x, \tau) \neq 0$;

(9) if $x \in \partial B_{4}^{c}$ then $\exists \tau \in[0, t]$ such that $\bar{q}(x, \tau) \neq 0$.

For the data of our mixed problem, we define the domain of influence at instant $t$ as follows:

$$
\mathcal{D}_{t}=\left\{\mathbf{x}_{0} \in \bar{B}: B(t) \cap \bar{\Gamma}\left(\mathbf{x}_{0}, v t\right) \neq \Phi\right\} .
$$

Here we denote by $\Phi$ the empty set.

Theorem 2 If the system of equations (12) admits a solution $\left(u_{i}, \varphi_{i j}, \phi, \theta\right)$ which satisfies the initial data (9) and the data at the limit (10), then for any $t>0$ we obtain

$$
u_{i}=0, \quad \varphi_{i j}=0, \quad \phi=0, \quad \theta=0 \quad \text { on }\left\{\bar{B} \backslash \mathcal{D}_{t}\right\} \times[0, t]
$$


Proof We will arbitrarily fix $\mathbf{x}_{0} \in \bar{B} \backslash \mathcal{D}_{t}$ and $\tau \in[0, t]$, and use inequality (17) taking $t=\tau$ and $\mathcal{R}=v(\tau-s)$. In this way, we are led to

$$
\begin{aligned}
& \int_{\Gamma\left[\mathbf{x}_{0}, v(\tau-s)\right]} U(\mathbf{x}, \tau) d V+\frac{1}{T_{0}} \int_{0}^{\tau} \int_{\Gamma\left[\mathbf{x}_{0}, v(\tau-s)\right]} k_{i j} \theta_{, i} \theta_{, j} d V d \alpha \\
& \leq \int_{\Gamma\left[\mathbf{x}_{0}, v \tau\right]} U(\mathbf{x}, 0) d V+\int_{0}^{\tau} \int_{\Gamma\left[\mathbf{x}_{0}, v(\tau-s)\right]} \varrho\left(F_{i} \dot{u}_{i}+M_{j k} \dot{\varphi}_{j k}+L \dot{\phi}+\frac{1}{T_{0}} r \theta\right) d V d \alpha \\
& \quad+\int_{0}^{\tau} \int_{\partial \Gamma\left[\mathbf{x}_{0}, v(\tau-s)\right]} \varrho\left(\bar{t}_{i} \dot{u}_{i}+\bar{\mu}_{j k} \dot{\varphi}_{j k}+\bar{h} \dot{\phi}+\frac{1}{T_{0}} \bar{q} \theta\right) d A d \alpha .
\end{aligned}
$$

Taking into account that $\mathbf{x}_{0} \in \bar{B} \backslash \mathcal{D}_{t}$, we deduce that $\mathbf{x} \in \Gamma\left[\mathbf{x}_{0}, v \tau\right]$ which involves that $\mathbf{x} \notin B(t)$. So, we deduce

$$
\int_{\Gamma\left[\mathbf{x}_{0}, v \tau\right]} U(\mathbf{x}, 0) d V=0
$$

Now, we take into account that $\Gamma\left[\mathbf{x}_{0}, v(\tau-s)\right] \subseteq \Gamma\left[\mathbf{x}_{0}, v \tau\right]$ in order to obtain the following two equalities:

$$
\begin{gathered}
\int_{0}^{\tau} \int_{\Gamma\left[\mathbf{x}_{0}, v(\tau-s)\right]} \varrho\left(F_{i} \dot{u}_{i}+M_{j k} \dot{\varphi}_{j k}+L \dot{\phi}+\frac{1}{T_{0}} r \theta\right) d V d \alpha=0, \\
\int_{0}^{\tau} \int_{\Gamma\left[\mathbf{x}_{0}, v(\tau-s)\right]}\left(\bar{t}_{i} \dot{u}_{i}+\bar{\mu}_{j k} \dot{\varphi}_{j k}+\bar{h} \dot{\phi}+\frac{1}{T_{0}} \bar{q} \theta\right) d V d \alpha=0 .
\end{gathered}
$$

Considering assumption (iii) and relations (32)-(34), we deduce that

$$
\int_{\Gamma\left[\mathbf{x}_{0}, v(\tau-s)\right]} U(\mathbf{x}, \tau) d V \leq 0
$$

From (38), by using inequality (16), we deduce

$$
\int_{\Gamma\left[\mathbf{x}_{0}, v(\tau-s)\right]} K(\mathbf{x}, \tau) d V \leq 0
$$

By using this inequality and considering the definition of function $K$, we are led to the following null values:

$$
\dot{u}_{i}\left(\mathbf{x}_{0}, \tau\right)=0, \quad \dot{\varphi}_{j k}\left(\mathbf{x}_{0}, \tau\right)=0, \quad \phi\left(\mathbf{x}_{0}, \tau\right)=0, \quad \theta\left(\mathbf{x}_{0}, \tau\right)=0
$$

for any $\left(\mathbf{x}_{0}, \tau\right) \in\left\{\bar{B} \backslash \mathcal{D}_{t}\right\} \times[0, t]$.

But $u_{i}\left(\mathbf{x}_{0}, 0\right)=0, \varphi_{j k}\left(\mathbf{x}_{0}, 0\right)=0$ for any $\mathbf{x}_{0} \in \bar{B} \backslash \mathcal{D}_{t}$, so we deduce that

$$
u_{i}\left(\mathbf{x}_{0}, \tau\right)=0, \quad \varphi_{j k}\left(\mathbf{x}_{0}, \tau\right)=0, \quad \phi\left(\mathbf{x}_{0}, \tau\right)=0, \quad \theta\left(\mathbf{x}_{0}, \tau\right)=0
$$

for any $\left(\mathbf{x}_{0}, \tau\right) \in\left\{\bar{B} \backslash \mathcal{D}_{t}\right\} \times[0, t]$, which ends the proof of Theorem 2 . 


\section{Conclusions}

We can conclude that the main result of the present paper is an extension of known SaintVenant's principle from classical elasticity in order to cover the theory of thermoelasticity of dipolar porous bodies. We have shown that the domain of influence theorem remains valid even if we exceeded the framework of classical mechanics.

Namely, the essence of the principle remains the same even if we have taken into consideration the effect of thermal treatment, the effect of dipolar structure, and the effect of voids.

\section{Funding}

Not applicable.

Availability of data and materials

Data sharing not applicable to this article as no datasets were generated or analysed during the current study.

\section{Competing interests}

The authors declare that they have no competing interests.

\section{Authors' contributions}

All authors contributed equally to the writing of this paper. All authors read and approved the final manuscript.

\section{Author details}

${ }^{1}$ Department of Mathematics and Computer Science, Transilvania University of Brasov, Brasov, Romania. ${ }^{2}$ Department of Mathematics, Texas A \& M University—Kingsville, Kingsville, USA. ${ }^{3}$ Florida Institute of Technology, Melbourne, USA. ${ }^{4}$ Department of Mathematics, Cankaya University, Ankara, Turkey. ${ }^{5}$ Institute of Space Sciences, Magurele, Romania.

\section{Publisher's Note}

Springer Nature remains neutral with regard to jurisdictional claims in published maps and institutional affiliations.

Received: 17 May 2018 Accepted: 3 July 2018 Published online: 13 July 2018

\section{References}

1. Nunziato, J.W., Cowin, S.C.: A nonlinear theory of materials with voids. Arch. Ration. Mech. Anal. 72, 175-201 (1979)

2. Cowin, S.C., Nunziato, J.W.: Linear elastic materials with voids. J. Elast. 13, 125-147 (1983)

3. Goodman, M.A., Cowin, S.C.: A continuum theory of granular material. Arch. Ration. Mech. Anal. 44, $249-266$ (1971)

4. lesan, D.: A theory of thermoelastic material with voids. Acta Mech. 60, 67-89 (1986)

5. Marin, M.: Weak solutions in elasticity of dipolar porous materials. Math. Probl. Eng. 2008, Article ID 158908 (2008)

6. Marin, M.: An approach of a heat-flux dependent theory for micropolar porous media. Meccanica 51(5), 1127-1133 (2016)

7. Eringen, A.C.: Theory of micromorphic materials with memory. Int. J. Eng. Sci. 10, 623-641 (1972)

8. Eringen, A.C.: Theory of thermo-microstretch elastic solids. Int. J. Eng. Sci. 28, 1291-1301 (1990)

9. Mindlin, R.D.: Micro-structure in linear elasticity. Arch. Ration. Mech. Anal. 16, 51-78 (1964)

10. Green, A.E., Rivlin, R.S.: Multipolar continuum mechanics. Arch. Ration. Mech. Anal. 17, 113-147 (1964)

11. Fried, E., Gurtin, M.E.: Thermomechanics of the interface between a body and its environment. Contin. Mech. Thermodyn. 19(5), 253-271 (2007)

12. Abbas, I.A.: A GN model based upon two-temperature generalized thermoelastic theory in an unbounded medium with a spherical cavity. Appl. Math. Comput. 245, 108-115 (2014)

13. Abbas, I.A.: Eigenvalue approach for an unbounded medium with a spherical cavity based upon two-temperature generalized thermoelastic theory. J. Mech. Sci. Technol. 28(10), 4193-4198 (2014)

14. Abbas, I.A., Abo-Dahab, S.M.: On the numerical solution of thermal shock problem for generalized magneto-thermoelasticity for an infinitely long annular cylinder with variable thermal conductivity. J. Comput. Theor. Nanosci. 11(3), 607-618 (2014)

15. Othman, M.I.A.: State space approach to generalized thermoelasticity plane waves with two relaxation times under the dependence of the modulus of elasticity on reference temperature. Can. J. Phys. 81(12), 1403-1418 (2003)

16. Sharma, J.N., Othman, M.I.A.: Effect of rotation on generalized thermo-viscoelastic Rayleigh-Lamb waves. Int. J. Solids Struct. 44(13), 4243-4255 (2007)

17. Othman, M.I.A., Hasona, W.M., Abd-Elaziz, E.M.: Effect of rotation on micropolar generalized thermoelasticity with two-temperatures using a dual-phase-lag model. Can. J. Phys. 92(2), 149-158 (2014)

18. Marin, M., Stan, G.: Weak solutions in elasticity of dipolar bodies with stretch. Carpath. J. Math. 29(1), 33-40 (2013)

19. Marin, M., Baleanu, D.: On vibrations in thermoelasticity without energy dissipati-on for micropolar bodies. Bound. Value Probl. 2016, Article ID 111 (2016)

20. Marin, M., Abbas, I.A., Kumar, R.: Relaxed Saint-Venant principle for thermoelastic micropolar diffusion. Struct. Eng Mech. 51(4), 651-662 (2014)

21. Carbonaro, B., Russo, R.: Energy inequalities in classical elastodynamics. J. Elast. 14, 163-174 (1984)

22. Chandrasekharaiah, D.S.: A uniqueness theorem in the theory of elastic. J. Elast. 18, 173-179 (1987)

23. Ignaczak, J., Carbonaro, B.: Domain of influence theorem in thermoelasticity with one relaxation time. J. Therm. Stresses 9, 79-91 (1986) 